\title{
Am Limit? - Einschätzungen zur Grundwasserbewirtschaftung im Großraum Grünstadt (Rheinland-Pfalz)
}

\author{
Frank Bitzer $^{1}$ (D) $\cdot$ Christoph Baumeister ${ }^{2}$ Jochen Kampf ${ }^{2} \cdot$ Martin Schykowski $^{2}$ \\ Eingegangen: 8. April 2021 / Überarbeitet: 21. Oktober 2021 / Angenommen: 3. Dezember 2021 / Online publiziert: 21. Januar 2022 \\ () Der/die Autor(en) 2022
}

\section{Zusammenfassung}

Im Großraum Grünstadt erfolgt die Wasserversorgung weitgehend dezentral aus Grundwasserleitern des Buntsandsteins. Dieser ist aufgrund der geologisch-tektonischen Verhältnisse räumlich komplex gegliedert. In Verbindung mit Abschätzungen zu Grundwasserfließrichtungen und Altersdatierungen ist eine Gliederung in bilanzierbare Teilräume möglich.

Daten zu Grundwasserständen und zur Grundwasserbeschaffenheit weisen auf mitunter erhebliche Veränderungen seit etwa 1960 hin. Daten zu den Grundwasserentnahmen liegen erst ab 2013 vor. Die annähernd konstanten Absenkungsraten können als Entwicklung in der späten instationären Phase langfristiger, gering schwankender Grundwasserentnahmen interpretiert werden.

Auf Basis der Wasserbilanz für den zentralen Bilanzraum wurde die dort zur Bilanzdeckung notwendige Grundwasserneubildung abgeschätzt. Sie wurde den Ergebnissen zweier methodisch unterschiedlicher Grundwasserneubildungsmodelle gegenübergestellt. Die dokumentierten Absenkungen lassen sich mit dem Neubildungsmodell auf Basis des flächendifferenzierten Bodenwasserhaushaltsmodells GWN-BW in Einklang bringen. Für den zentralen bilanzierten Teilraum ist von einer langfristig über der Grundwasserneubildung liegenden Grundwasserentnahme auszugehen.

Schlüsselwörter Buntsandstein · Hydrogeologisches Modell · Grundwasserneubildung

\section{Off limit?-Assessments of groundwater management in the Grünstadt area (Rhineland-Palatinate)}

\begin{abstract}
In the Grünstadt area the water supply is realised by mostly local pumping wells in the Bunter aquifer system. The Bunter has a spatially complex structure due to the geological-tectonic setting. In combination with estimations to the groundwater flow direction and groundwater age, the area can be split into balanceable sub-areas.

Data of groundwater levels and hydrochemistry indicate significant changes since around 1960. Data on groundwater pumping are only available from 2013. The almost constant groundwater subsidence rates can be interpreted as a development in the late transient phase of long-term, slightly fluctuating groundwater pumping.

On the basis of the water balance for the central area, the groundwater recharge required to fit the balance was estimated. It was compared to the results of two methodologically different groundwater recharge models. The documented groundwater subsidence fits with the recharge model based on the area-differentiated soil water balance model GWN-BW. For the central area, long-term groundwater pumping above the recharge can be assumed.
\end{abstract}

Keywords Bunter $\cdot$ Hydrogeological model $\cdot$ Groundwater recharge

\author{
Frank Bitzer \\ frank.bitzer@lgb-rlp.de \\ Christoph Baumeister \\ christoph.baumeister@1fu.rlp.de \\ Jochen Kampf \\ jochen.kampf@lfu.rlp.de
}

\author{
Martin Schykowski \\ martin.schykowski@lfu.rlp.de \\ 1 Landesamt für Geologie und Bergbau Rheinland-Pfalz, \\ Emy-Roeder-Straße 5, 55129 Mainz, Deutschland \\ 2 Landesamt für Umwelt Rheinland-Pfalz, \\ Kaiser-Friedrich-Straße 7, 55116 Mainz, Deutschland
}




\section{Einleitung}

Die Trinkwasserversorgung in Rheinland-Pfalz erfolgt zu ca. 95\% durch Grundwasserförderung (Kampf et al. 2007). Die mesozoischen Senken mit weitflächiger Verbreitung von mächtigem Buntsandstein (Trier-Bitburger Senke und Pfälzer Mulde) sowie die Lockergesteinsfüllung des Oberrheingrabens stellen hierbei die bedeutendsten großräumigen Grundwasservorkommen dar (Ad-hoc-Ag Hydrogeologie 2016; Kampf und Schwebler 2005; s. Abb. 1a).

\section{Untersuchungsraum Grünstadt}

Der Raum Grünstadt befindet sich im südlichen RheinlandPfalz. Er liegt geologisch gesehen im Übergangsbereich des nordwestlichen Oberrheingrabes, des Mainzer Beckens, der Pfälzer Mulde als südliche mesozoische Senke und dem Permokarbon des Pfälzer Sattels (als südöstlichem Teil der paläozoischen Senken; Abb. 1a).

Mit etwa 110.000 Einwohnern, intensiver landwirtschaftlicher Nutzung und diverser Industrie belief sich der Wasserbedarf im Jahr 2014 im Untersuchungsgebiet auf etwa 6,7 Mio. $\mathrm{m}^{3} / \mathrm{a}$.

$\mathrm{Zu}$ diesem Zeitpunkt erfolgte die Wasserversorgung durch 10 Versorgungsunternehmen mit etwa 50 Brunnen, die das Grundwasser fast ausnahmslos aus dem Grundwasserleitersystem des Buntsandsteins fördern. Die räumliche Verteilung bzw. ungefähre Lage der Brunnen ist in der Abb. 1b dargestellt. Das Konzept der Grundwasserbewirtschaftung war bis zum o.g. Zeitpunkt ausschließlich auf die einzelnen Gewinnungsgebiete bezogen. Eine zusammenhängende, großräumige Betrachtung der Grundwasserförderung aus dem Buntsandsteinaquifer bestand ebenso wenig, wie eine zu diesem Zweck geeignete systematische Grundwasserbeobachtung mittels Messstellen.

\section{Untersuchungsumfang}

Im Rahmen der Untersuchungen erfolgte eine komplette geologisch-tektonische Neubearbeitung des Raums. Diese beinhaltete eine Überarbeitung der bisher vorliegenden Kartenblätter aus dem Zeitraum seit 1913 unter Berücksichtigung des aktuellen Bestands der Bohrungsinformationen sowie geophysikalischer Untersuchungsergebnisse aus verschiedenen Kampagnen.

Auf diese grundlegenden Arbeiten wird im Folgenden nicht weiter eingegangen und auf die Veröffentlichungen von Baumeister et al. (2016) und Bitzer (2012) verwiesen.

Aufgrund von Unvollständigkeit der von der Wasserwirtschaft (Struktur- und Genehmigungsdirektionen als obere Wasserbehörden) erhobenen Daten zu Grundwasserständen und Grundwasserbeschaffenheit in den Gewinnungsgebieten wurden diese Informationen im Rahmen einer aufwendigen Recherche bei den Wasserversorgern ergänzt. Sie liegen nun in unterschiedlichster Form i. W. für den Zeitraum von etwa 1963 bis 2010 vor. Daten aus dem Landesmessnetz (betrieben vom Landesamt für Umwelt) gibt es nur für wenige, i. W. außerhalb der Gewinnungsgebiete liegende Punkte und beziehen sich v.a. auf den oberen Grundwasserleiter.

Im Resultat konnten für einige Gewinnungsgebiete Zeitreihen unterschiedlicher Auflösung zu Grundwasserständen und Grundwasserbeschaffenheit für den Buntsandsteinaquifer erstellt werden (Bitzer 2013). Diese werden hier auszugsweise vorgestellt (s. a. Abb. 4).

Belastbare Daten zu den Grundwasserentnahmen liegen bei der für die Wasserwirtschaft zuständigen Strukturund Genehmigungsdirektion Süd (Regionalstelle Neustadt) erst mit der Einführung des Entnahmeentgeltgesetzes (LWEntG) ab dem 01.01.2013 vor. Für den davorliegenden Zeitraum wurden ab 1995 nur von wenigen Wasserversorgern zeitweise Daten auf freiwilliger Basis mitgeteilt (Abb. 2). Zeitlich gesehen sind die Entnahmedaten deutlich jünger als die erhobenen Daten zu Grundwasserständen und Grundwasserbeschaffenheit. Die Jahresentnahmen aus dem Buntsandsteinaquifer seit 2013 weisen nur geringe Schwankungen auf.

Auf der Basis der zuvor skizzierten Arbeiten wurden ausgewählte Wasserfassungen zur Klärung der Altersstruktur des Grundwassers im Buntsandsteinaquifer beprobt. Zum Einsatz kamen Methoden zur Messung und Interpretation der Gehalte von $\mathrm{SF}_{6}, \mathrm{FCKW},{ }^{13} \mathrm{C} /{ }^{14} \mathrm{C},{ }^{16} \mathrm{O}$ und ${ }^{2} \mathrm{H}$. Die Analytik erfolgte durch das Spurenstofflabor Dr. Oster (Wachenheim) und die Hydroisotop GmbH (Schweitenkirchen). Wesentliche Ergebnisse der Untersuchungen werden im Folgenden vorgestellt (s. a. Abb. 3).

Auf dieser Informationsbasis beruhend wurde ein hydrogeologisches Modell für den Untersuchungsraum erstellt. Ziel war es dabei, eine Gliederung in bilanzierbare Teilräume zu entwickeln. Die beobachteten zeitlichen Entwicklungen von Grundwasserständen und der Grundwasserbeschaffenheit wurden für bilanzierbare Teilräume unter Nutzung der verfügbaren Daten zu Grundwasserentnahmen und der Grundwasserneubildung interpretiert.

Auf das hydrogeologische Modell, die Abgrenzung des zentralen bilanzierbaren Teilraums und die Einordnung der Entwicklung von Grundwasserständen und Grundwasserbeschaffenheit wird im Folgenden eingegangen. 


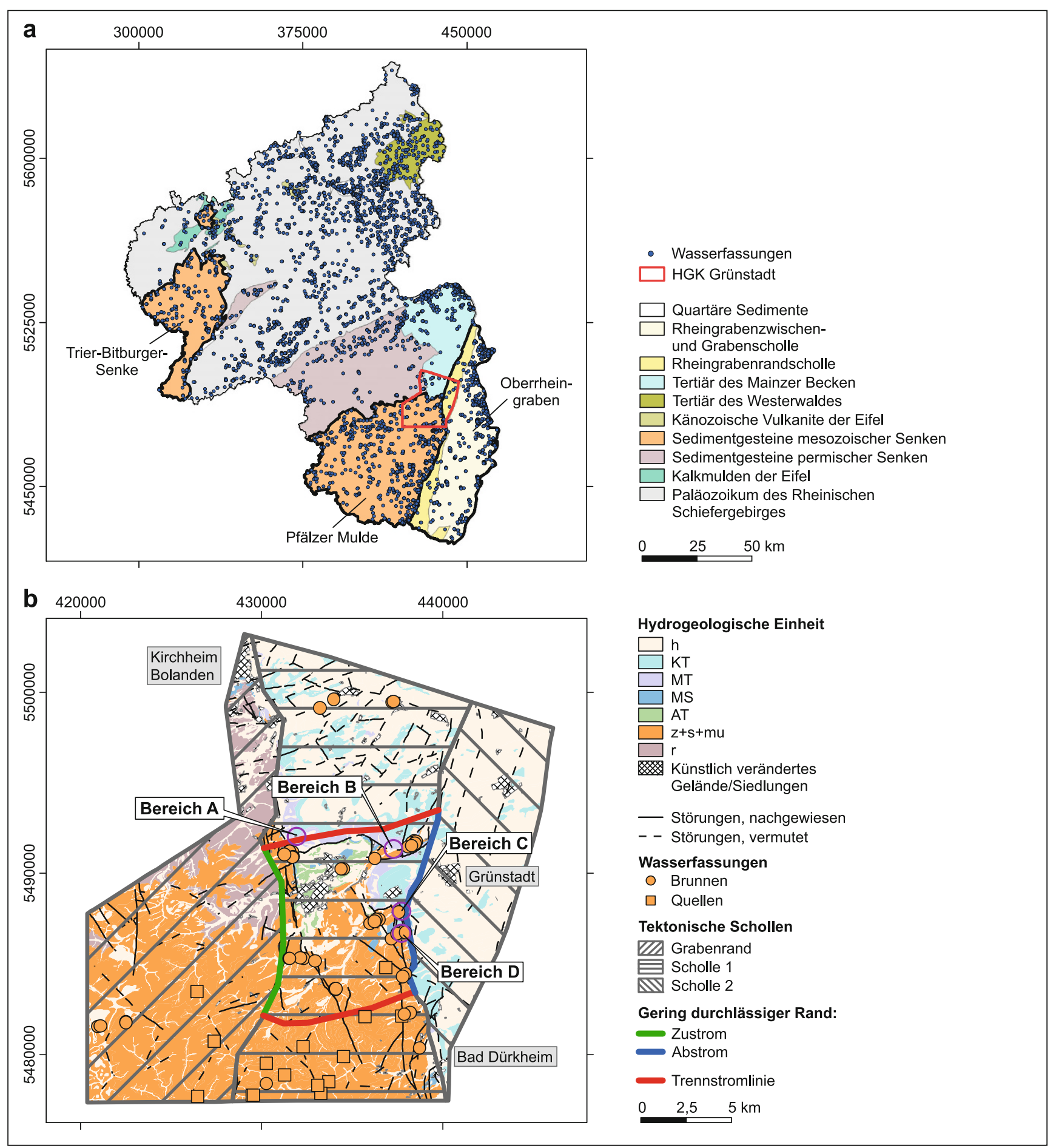

Abb. 1 a Hydrogeologische Raumgliederung (vereinfacht nach BGR 2015) und Grundwasserentnahmestellen in Rheinland-Pfalz. Fett umrandet: Trier-Bitburger Senke, Pfälzer Mulde und Oberrheingraben; rot umrandet: Untersuchungsraum „HGK Grünstadt“; b Vereinfachte Karte der hydrogeologischen Einheiten und tektonischen Gliederung (vereinfacht nach Baumeister et al. 2016) sowie Grundwasserentnahmestellen im Buntsandstein (Brunnen und Quellen); A bis D: Bereiche signifikanter Veränderungen der Grundwasserverhältnisse (Erläuterungen im Text, s. a. Abb. 4). Im Zentrum: Hydraulische Ränder des Bilanzraums

Fig. 1 a Hydrogeological spatial structure of Rhineland-Palatinate (BGR 2015, simplified) and places of groundwater extraction. Bold outlined: Trier-Bitburg Syncline, Palatinate Syncline and Upper Rhine Graben; red outlined: area of interest „HGK Grünstadt“; b Map of the hydrogeological units and tectonic structure (Baumeister et al. 2016, simplified) and places of groundwater extraction in the Bunter (wells and springs). A to D: areas of significant changes in the groundwater conditions (for details see text, s. a. Abb. 4). Center: hydraulic boundaries of the balance area 
Abb. 2 Jahresentnahmen im Untersuchungsgebiet (räumlich gegliedert). Die höchsten Entnahmen erfolgen im zentralen Bereich (vgl. Abb. 1b). Belastbar sind die Daten erst ab dem Jahr 2013

Fig. 2 Annual groundwater extraction (spatially structured, s. a. Abb. 1b). Highest pumping rates are realised in the central area. The data are only reliable from 2013 onwards

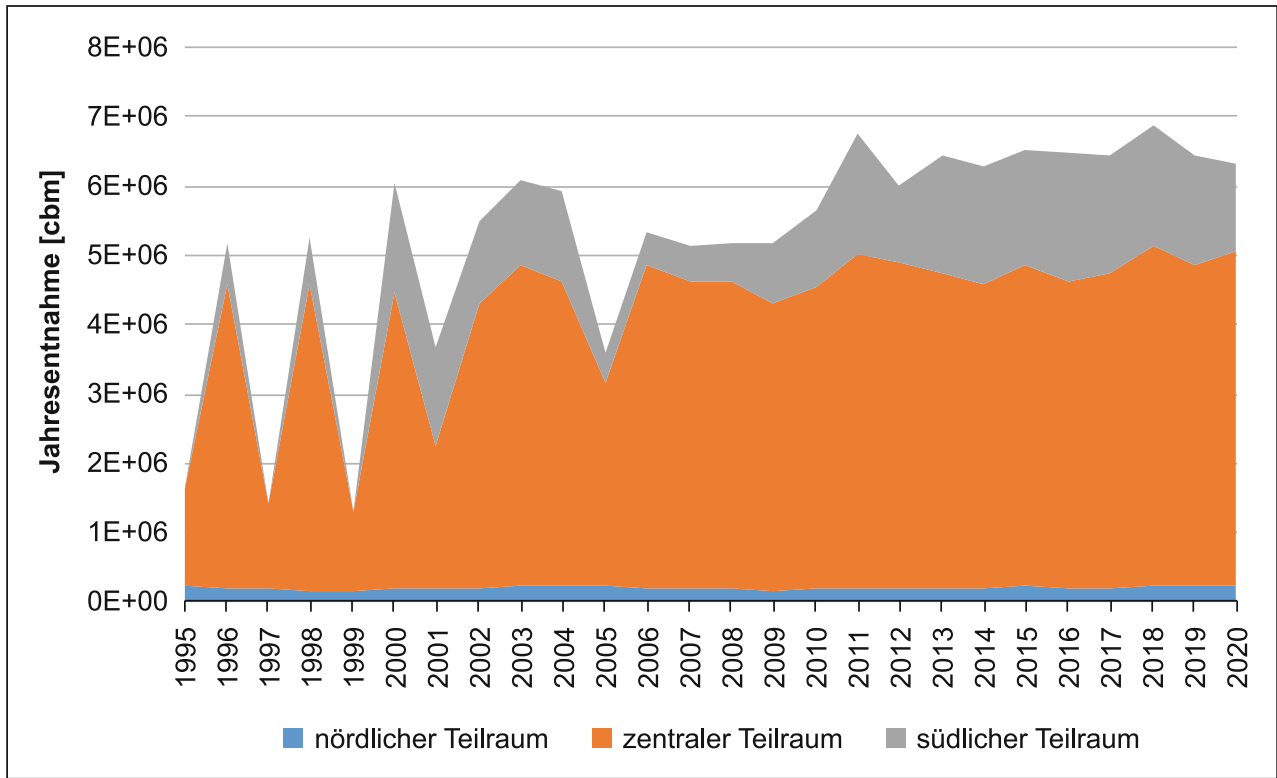

\section{Hydrogeologisches Modell - räumliche Gliederung}

Das bis zu $450 \mathrm{~m}$ mächtige Grundwasserleitersystem des Buntsandsteins ist gekennzeichnet durch je nach Schichtfolge wechselnde kluft- und porengestützte Hohlraumanteile. Zusammen mit dem geringmächtigen unterlagernden Zechstein und lokal reliktisch erhaltenem Unteren Muschelkalk im Hangenden bildet es die wasserwirtschaftlich bedeutsame hydrogeologische Einheit $\mathrm{z}+\mathrm{s}+\mathrm{mu}$ (s. Abb. 1b).

Vom zentralen bis zum nördlichen Bereich des Untersuchungsraums wird der Buntsandstein durch tertiäre Ablagerungen überdeckt. Diese werden unterschieden in die hydrogeologischen Einheiten AT (Alttertiär), MS (Meeressand), MT (Mergeltertiär) und KT (Kalktertiär, als jüngste Einheit).

Die flächenhaft verbreitete Einheit MT bewirkt (zusammen mit der lokal verbreiteten Einheit AT) aufgrund der sehr geringen mittleren Gebirgsdurchlässigkeiten eine hydraulisch streng wirksame Grundwasserstockwerkstrennung zwischen der Einheit $\mathrm{z}+\mathrm{s}+\mathrm{mu}$ im Liegenden (sowie der bereichsweise auflagerndem Einheit MS) und der Einheit KT im Hangenden.

Im südlichen Bereich bildet die Einheit $\mathrm{z}+\mathrm{s}+\mathrm{mu}$ das obere Grundwasserstockwerk.

Die geringmächtige Einheit h (quartäre Lockersedimente) im Norden und Osten besteht aus den jüngsten Ablagerungen.

Die den Oberrheingraben flankierenden Störungssysteme gliedern den Buntsandstein von Westen nach Osten in drei treppenartig abgesunkene Schollen, die intern weiter segmentiert sind. In der Gliederung und Bezeichnung der
Schollen wird der Systematik von Franke und Bitzer (2019) gefolgt (s. Abb. 1b).

Auf der Grabenrand-Scholle, d.h. westlich der Scholle 1, fallen die Schichten des Buntsandsteins und Zechsteins generell in südliche Richtung ein.

Die Mächtigkeit des Buntsandsteins erreicht im Süden bis etwa $100 \mathrm{~m}$, am Grabenrand selbst jedoch nur wenige 10er Meter. Im zentralen und nördlichen Bereich fehlt der Buntsandstein.

Im Bereich der Scholle 1 (die nach Norden hin Bereiche des Mainzer Beckens umfasst; s. a. Abb. 1) ist das Schichteinfallen des Buntsandsteins und Zechsteins nach Norden gerichtet.

Der Buntsandstein erreicht im zentralen Bereich bis etwa $450 \mathrm{~m}$ Mächtigkeit; nach Norden hin keilt er etwa am nördlichen Rand des Untersuchungsgebiets aus. Im Süden erreicht er Restmächtigkeiten von z. T. nur um $100 \mathrm{~m}$.

Im Bereich der Scholle 2 steht der Buntsandstein nicht obertägig an. Die Mächtigkeit des überlagernden Tertiärs (unter geringmächtigen quartären Deckschichten) wird dort mit etwa $200 \mathrm{~m}$ vermutet und der Top Buntsandstein wird bei etwa 0 bis $-100 \mathrm{mNN}$ angenommen (Franke und Bitzer 2019; Baumeister et al. 2016).

\section{Grundwasserströmung und Altersstruktur}

Die Grundwasserströmungsverhältnisse innerhalb der hydrogeologischen Einheit $z+s+m u$ wurden abgeleitet auf der Basis von Grundwasserstandsdaten verschiedenen Messdatums und unter Berücksichtigung der jeweiligen Ausbausituation. Hierbei wurden ebenfalls die angrenzenden hydrogeologischen Einheiten r (Rotliegend) und KT 
Abb. 3 Grundwassergleichen im Buntsandstein-Aquifersystem sowie hydraulisch angeschlossener Einheiten (konzeptionelles Modell). Grundwasserbildungsalter $\left({ }^{14} \mathrm{C}\right.$-Methode) und prozentuale Abschätzung von Jungwasseranteilen $\left(\mathrm{SF}_{6}\right.$ und FCKW-Methode)

Fig. 3 Isolines of the groundwater heads in the Bunter aquifer system and hydraulically connected units (conceptional model). Age of groundwater formation $\left({ }^{14} \mathrm{C}\right.$-method) and estimated percentage of the young water component $\left(\mathrm{SF}_{6}\right.$ und FCKW-method)

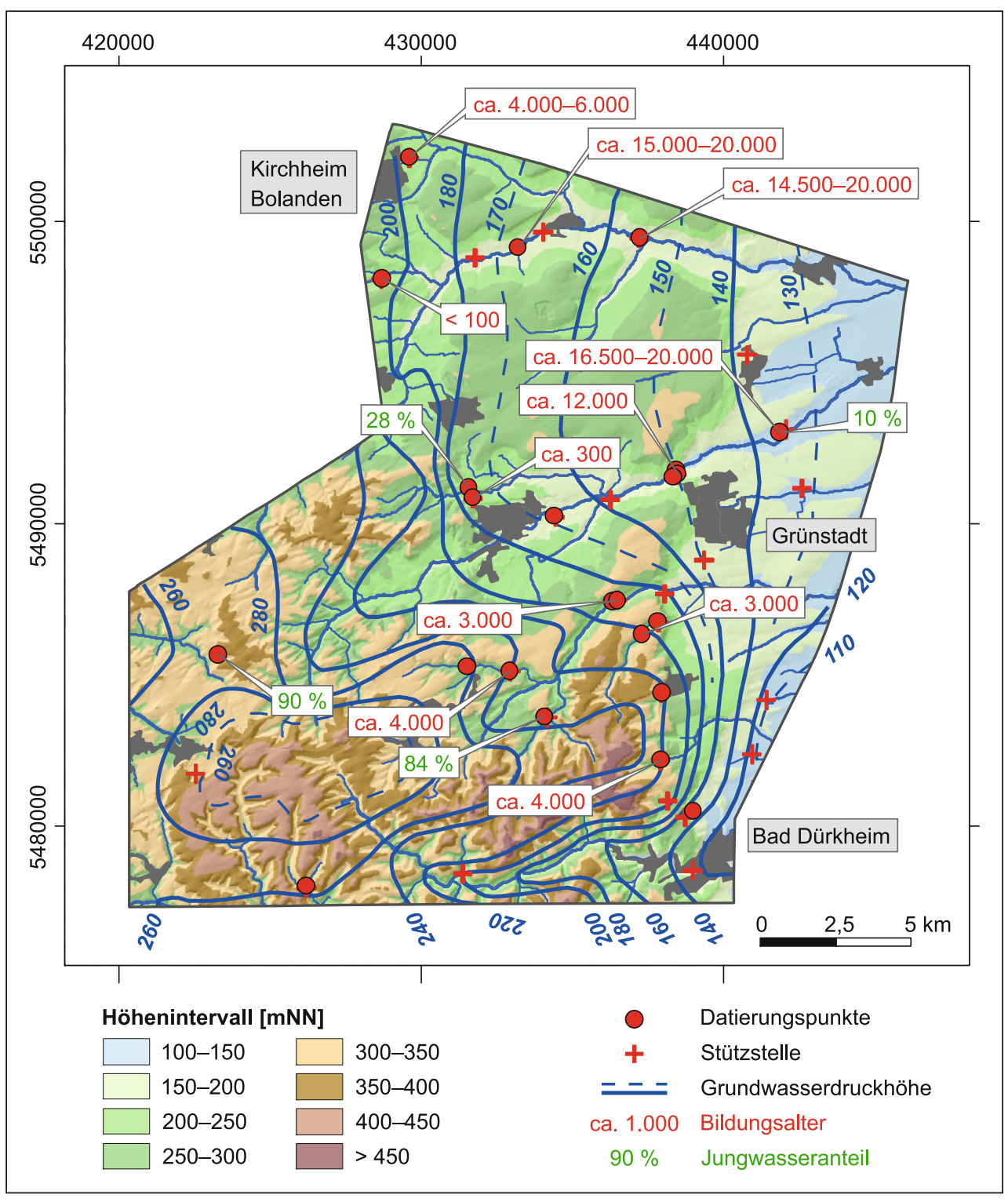

(Kalktertiär) sowie Gewässerhöhen, Abflussverhältnisse und Höhen von Quellaustritten im Verbreitungsbereich der Einheit $\mathrm{z}+\mathrm{s}+\mathrm{mu}$ berücksichtigt. Im Ergebnis entstand ein konzeptioneller Gleichenplan der Grundwasserdruckhöhen (Abb. 3).

Im nördlichen und zentralen Bereich des Untersuchungsgebiets nehmen die Grundwasserdruckhöhen im Buntsandsteinaquifer relativ gleichmäßig von Westen nach Osten ab. Aufgrund der hier bestehenden Grundwasserstockwerkstrennung ist die Druckhöhenverteilung im Wesentlichen unbeeinflusst von dort verlaufenden Gewässern. Die Grundwasserströmung ist großräumig nach Osten in Richtung Rhein orientiert.

Im südlichen Bereich ändert sich das Bild: Die Druckhöhenverteilung (Buntsandstein hier oberes Grundwasserstockwerk) ist wesentlich durch das Gewässernetz und die
Topografie beeinflusst. Entsprechend ist im Süden auch eine etwa Ost-West verlaufende Wasserscheide ausgebildet.

Die zur Klärung der Altersstruktur in der hydrogeologischen Einheit $\mathrm{z}+\mathrm{s}+\mathrm{mu}$ angewendeten Methoden zielten auf die Abschätzung von Jungwasseranteilen (jünger etwa 10 Jahre), mittleren Verweilzeiten sowie Bildungsaltern (Grundwasseraltersabschätzung mit dem ${ }^{14} \mathrm{C}$-Isotop) des Grundwassers.

Die abgeleiteten Bildungsalter im Bereich der Scholle 1 nehmen von Süden nach Norden zu und erreichen bis etwa 20.000 Jahre. Die Bildungsalter steigen im zentralen Bereich ebenfalls von Westen nach Osten auf Alter von etwa 12.000 bis 20.000 Jahre an (Abb. 3); Jungwasseranteile sind nicht vorhanden.

Gleichzeitig sind die Jungwasseranteile in den südlich, topografisch höher gelegenen Bereichen sowie im Nahbe- 
reich zum östlich anschließenden Permokarbon des Pfälzer Sattels am höchsten. Im zentralen Bereich sowie in östliche Richtung nehmen sie ab.

Die Ergebnisse zur Altersstruktur des Grundwassers fügen sich gut in das Bild des konzeptionellen Gleichenplans und der festgestellten Grundwasserstockwerksgliederung ein. Anhand der abgeschätzten Grundwasserströmungsverhältnisse, der ermittelten Jungwasseranteile und der Bildungsalter ist zu erkennen, dass das Grundwasserneubildungsgebiet der hydrogeologischen Einheit $\mathrm{z}+\mathrm{s}+\mathrm{mu}$ vor allem im südlichen Bereich der Scholle 1 liegt (Baumeister et al. 2016). Mit zunehmendem Fließweg und insbesondere in den durch die Stockwerksgliederung geprägten Bereichen steigen die Bildungsalter erheblich an, Jungwasseranteile hingegen fehlen.

Auf Basis des konzeptionellen Gleichenplans und der Kenntnisse zur räumlichen, hydrogeologischen Gliederung war es möglich, Trennstromlinienbereiche zu definieren, die den zentralen Untersuchungsraum im Bereich der Scholle 1 nach Norden und Süden hin begrenzen (Abb. 1b).

Weiterhin können entlang der tektonisch bedingten Grenzen und dem damit verbundenen Aneinandergrenzen unterschiedlicher hydrogeologischer Einheiten $\mathrm{Zu}$ - und $\mathrm{Ab}$ stromränder im Westen und Osten des zentralen Untersuchungsraums abgeleitet werden (s. Abb. 1b). Damit besteht die Möglichkeit einer Grundwasserbilanzierung für diesen zentralen Bereich.

\section{Zeitliche Entwicklung von Grundwasserständen und Grundwasserbeschaffenheit - die Suche nach Einflussfaktoren}

Im Folgenden werden Daten zur zeitlichen Entwicklung von Grundwasserständen und der Grundwasserbeschaffenheit für den zentralen, bilanzierbaren Bereich der Scholle 1 vorgestellt (Bereiche A bis D, s. Abb. 1b). Da keine durch die obere Wasserbehörde (Struktur- und Genehmigungsdirektion) koordinierte Erfassung dieser Daten erfolgte, waren diese nur bei wenigen Wasserversorgern für unterschiedliche Zeiträume und in verschiedenster zeitlicher Auflösung recherchierbar.

Der Bereich A (Abb. 1b und 4) umfasst die Gewinnungsgebiete „Ei““ und „Gö“. Sie liegen am westlichen Rand der Scholle 1, etwa in Höhe des zentralen Untersuchungsgebiets (vgl. Abb. 1b). Die bei Brunnenbohrungen angetroffenen Wasserstände weisen in der zeitlichen Abfolge die Charakteristik der Absenkung bei einem Pumpversuch auf. Die Zeitachse reicht vom Jahr 1963 bis 1997. Der Wasserspiegel ist in diesem Intervall um etwa $36 \mathrm{~m}$ (nicht linear) gesunken; für den jüngeren Zeitraum von 1988 bis 1997 ergibt sich eine mittlere, quasi-konstante Absenkungsrate von etwa $0,1 \mathrm{~m} / \mathrm{a}$. Entnahmedaten für diesen Zeitraum liegen nicht vor.

Bereich B umfasst eine etwa $1 \mathrm{~km}$ lange Brunnengalerie in einem Taleinschnitt, die nur dort den Buntsandstein lokal als erstes Grundwasserstockwerk erschließt. Auf den umgebenden Plateauflächen hingegen ist das Tertiär (hydrogeologische Einheit KT; s. a. Abb. 1b) als erstes Grundwasserstockwerk ausgebildet. Im Hangbereich wird es durch zwei Quellen erschlossen. Die Einheit MT trennt die beiden Grundwasserstockwerke.

Im Beobachtungszeitraum zeigen alle Wasserfassungen (außer Brunnen 13) tendenziell zunehmende elektrische Leitfähigkeiten. Die höchsten Werte weisen die beiden Quellen (beide Einheit KT) und die flach abgesperrten Brunnen 6, 7 und 8 auf (alle Einheit $z+s+m u$ ). In den Hangbereichen austretendes kalktertiäres Grundwasser sickert offenbar dem im Tal anstehenden Buntsandstein zu und erhöht bei gleichzeitig fallenden Grundwasserdruckhöhen dessen elektrische Leitfähigkeit im oberflächennahen Bereich. In den flach abgesperrten Brunnen steigen die elektrischen Leitfähigkeiten.

Die tiefer abgesperrten Brunnen 9, 10 und 11 (alle Einheit $z+s+m u)$ zeigen diesen Trend in abgeschwächter Form. Die Grenzfläche des durch kalktertiäres Grundwasser beeinflussten Buntsandsteingrundwassers sinkt mit der Zeit offenbar in tiefere Niveaus und beeinflusst auch dort die ursprüngliche Beschaffenheit.

Lediglich der Brunnen 13 mit einem Erschließungsbereich von etwa 185 bis $316 \mathrm{~m}$ unter Gelände erfasst reines Buntsandstein-Grundwasser (Bildungsalter etwa 12.000 Jahre) und ist unbeeinflusst von dieser Entwicklung.

Die Entnahmen im dargestellten Zeitraum betrugen zwischen 1,4 und 1,7 Mio. m³/a. Die Entnahmekonfiguration, d.h. räumliche Verteilung der Entnahmen und die Höhe der Einzelentnahmen auf die einzelnen Brunnen blieben in diesem Zeitraum weitgehend unverändert.

Bereich $\mathrm{C}$ befindet sich am östlichen Rand der Scholle 1, etwa in Höhe des zentralen Untersuchungsgebiets (vgl. Abb. 1b). Die ursprünglich zur Altlastenüberwachung eingerichtete Messstellengruppe ist zwischen 1 bis $1,5 \mathrm{~km}$ von zwei benachbarten Gewinnungsgebieten entfernt. In diesen lag die Gesamtentnahmerate im dargestellten Zeitraum bei etwa 1,4 Mio. $\mathrm{m}^{3} / \mathrm{a}$. Es bestehen keine näherliegenden Grundwasserentnahmen. Im Zeitraum der Jahre 1995 bis 2010 fällt der Grundwasserstand annähernd stetig bis zu $0,5 \mathrm{~m} / \mathrm{a}$.

Bereich D liegt etwa $1 \mathrm{~km}$ südlich des Bereichs $\mathrm{C}$, ebenfalls an der östlichen Grenze der Scholle 1 (Abb. 1b). Abb. 4 zeigt die Grundwasserstandsentwicklung in den Messstellen GWM 1 bis 3, die den drei Brunnen unmittelbar benachbart sind, sowie in der etwa $800 \mathrm{~m}$ entfernten Messstelle GWM 4. Mit Beginn der Grundwasserentnahmen Mitte 
Abb. 4 Entwicklung von Grundwasserständen (Bereiche A, C, D; verschiedene Zeitintervalle). Entwicklung der elektrischen Leitfähigkeit (Bereich B; Quellen: oberes Grundwasserstockwerk, hydrogeologische Einheit KT; Brunnen: zweites Grundwasserstockwerk, Entnahmetiefe Brunnen 13: 185 bis $315 \mathrm{~m}$ uGOK; hydrogeologische Einheit $\mathrm{z}+\mathrm{s}+\mathrm{mu})$. Zur Lage der Bereiche A bis D siehe Abb. $1 b$ Fig. 4 Times series of groundwater levels (areas A, C, D; different time intervals). Times series of electrical conductivity (area B; springs: upper groundwater level, hydrogeological unit $\mathrm{KT}$; wells: second groundwater level, depth of groundwater extraction in well 13: 185 to $315 \mathrm{~m}$ below ground; hydrogeological unit $z+s+m u$ ). For location of the areas A to D see Abb. 1b

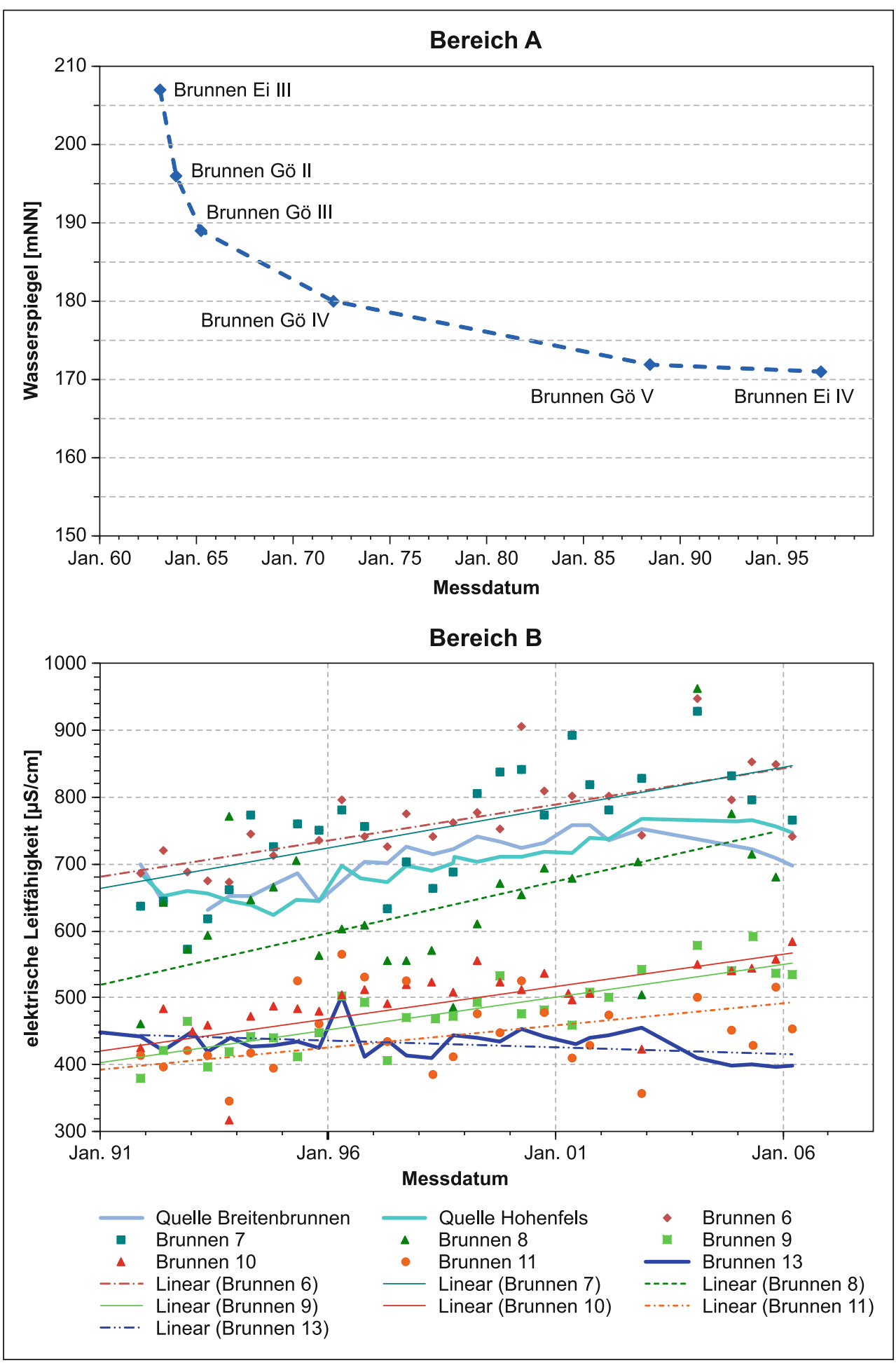

1994 fallen die nah gelegenen Messstellen bis 1997 trocken. Die entfernt gelegene GMW 4 spiegelt die saisonale, niederschlagsbedingte Entwicklung der Grundwasserstände wider. Ihr Verlauf ist jedoch geprägt durch den überlagernden Trend stetig fallender Grundwasserstände, der durch die benachbarte Grundwasserentnahme bedingt ist. Bis etwa 14 Jahre nach Inbetriebnahme des Gewinnungs- gebiets besteht eine annähernd konstante Absenkungsrate von 1,4 m/a. Die Messstelle GWM 4 ist im Jahr 2010 bei einem Grundwasserstand von etwa $43 \mathrm{~m}$ unter Gelände trockengefallen.

Die Entnahmen im dargestellten Zeitraum lagen bei weitgehend konstanter Entnahmekonfiguration zwischen 0,8 und 1,0 Mio. $\mathrm{m}^{3} / \mathrm{a}$. 
Abb. 4 (Fortsetzung)

Fig. 4 (continued)

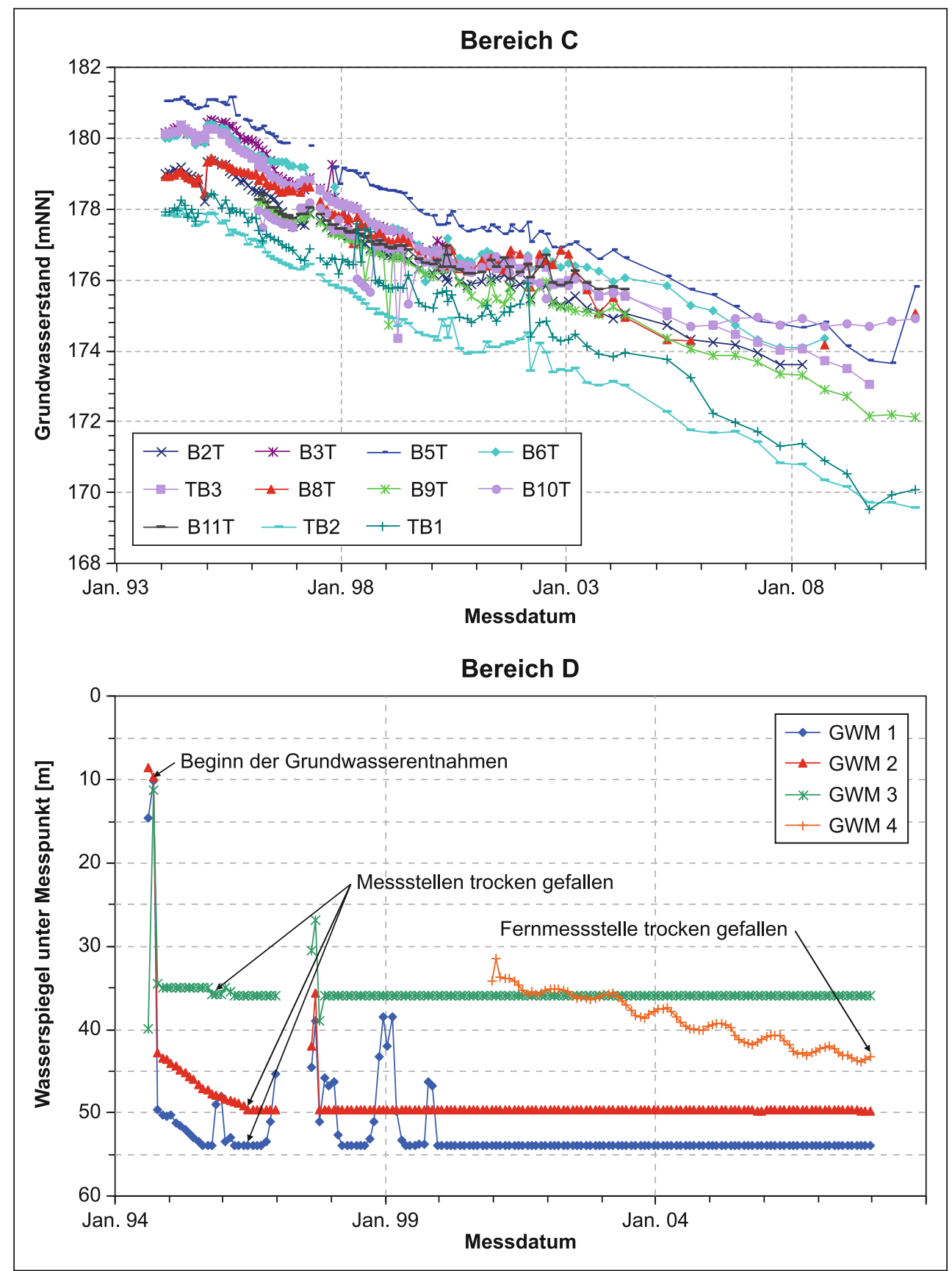

Sowohl in den Randgebieten der Scholle 1 (Bereiche A, $\mathrm{C}$ und D) als auch in deren Zentrum (Bereich B) lassen sich in der hydrogeologischen Einheit $z+s+m u$ im Zeitraum von 1963 bis 2010 der Trend fallender Grundwasserstände bzw. Veränderungen der Grundwasserbeschaffenheit als Folgewirkung erkennen. Im Bereich A ist die Entwicklung der Grundwasserstände entsprechend einem über 30 Jahre andauernden Pumpversuch dokumentiert.

Insbesondere die Abnahme der Grundwasserdruckhöhen erfolgte in den Beobachtungszeiträumen (mit Ausnahme der relativ frühen Phase im Bereich $\mathrm{A}$ ) annähernd linear und erinnert damit an die relativ späte Phase eines Pumpversuchs. Der zeitliche Verlauf der Druckhöhen (im Trend linear und quasi stetig abnehmend) lässt auf eine zumindest in den Beobachtungszeiträumen relativ konstante Entnahmesituation in den Bereichen B bis D schließen. Leider fehlen die Daten zur zeitlichen und räumlichen Entwicklung der Entnahmemengen im Untersuchungsgebiet für die dargestellten Zeiträume. Die aufgezeigten Entwicklungen weisen auf eine langandauernde Übernutzung des Buntsandsteinaquifers hin. 
Für die Zukunft ist eine Überlagerung der skizzierten Entwicklungen durch ungünstige klimatische Änderungen zu erwarten. Auf Basis der landesweit ausgewerteten Daten des Rheinland-Pfalz Kompetenzzentrums für Klimawandelforschung (2019) sind im Vergleich zu den Mittelwerten des Zeitraums 1971 bis 2000 seit den 1990er-Jahren folgende Veränderungen festzustellen:

- gestiegene Jahresdurchschnittstemperaturen (um etwa $\left.1,1^{\circ} \mathrm{C}\right)$ und

- gesunkene Jahresniederschlagshöhen (seit 2010 bis zu $20 \%)$.

Damit verbunden kam es bei Kulturpflanzen im Zeitraum 1988 bis 2017 (bzw. 1989 bis 2018) zu einer Verlängerung der Vegetationsperiode gegenüber dem langjährigen Mittel (Zeitraum 1951 bis 1980) um 14 (bzw. 18) Tage im Jahr (Reiter et al. 2018). Bereits aus diesen Veränderungen ist eine verringerte Grundwasserneubildung zu folgern.

Aufgrund der eingetretenen klimatischen Veränderungen wächst der Bedarf an verlässlichen Abschätzungen zur Grundwasserneubildung als Basis für eine nachhaltige Grundwasserbewirtschaftung.

\section{Grundwasserneubildung und Grundwasserbilanzierung}

Die Grundwasserneubildung ist die wesentliche positive Grundwasserbilanzkomponente. Ihre Abhängigkeit von den klimatischen Rahmenbedingungen und deren Auswirkungen auf die Sicherstellung der Wasserversorgung ist bundesweit zum Thema geworden (z. B. Bielitz und Winkler 2019; Wiesner und Bogel 2019). Bei der Nutzung von Grundwasserressourcen ist die verlässliche Abschätzung der Grundwasserneubildung Voraussetzung für die Entwicklung von Bewirtschaftungskonzepten (Mueef 2019).

Für das Untersuchungsgebiet wurden von Baumeister et al. (2016) zwei methodisch unterschiedliche Varianten zur Abschätzung der Grundwasserneubildung miteinander verglichen.

\section{Variante I}

Unter der Annahme, dass Gewässer in Trockenwetterperioden ausschließlich von Grundwasser gespeist werden, kann aus dem Trockenwetterabfluss und der Fläche des dazugehörigen Einzugsgebiets auf die Grundwasserneubildung geschlossen werden. Berechnet wurden die Abflusswerte nach dem KILLE-Verfahren. Die Grundwasserneubildungshöhen werden nach hydrologischen und hydrogeologischen Gesichtspunkten in die Kleinsteinzugsgebiete übertragen (Baumeister und Kampf 2002).
Auf diesem Wege wurden die mittleren natürlichen Grundwasserneubildungshöhen für den Zeitraum der Jahre 1979 bis 2008 abgeschätzt (Abb. 5). Diese Grundwasserneubildungshöhen sind auf dem geoportal-wasser.rlp in dem jeweils verfügbaren Stand veröffentlicht und abrufbar.

\section{Variante II}

Das Simulationsmodell GWN-BW schätzt als deterministisches, flächendifferenziertes Bodenwasserhaushaltsmodell zunächst die Sickerwassermenge ab. Über den sog. Baseflow-Index (BFI) ist die Abtrennung des neubildungsrelevanten Anteils möglich.

Die tatsächliche Verdunstung als Funktion vor allem von Temperatur, Niederschlag, Bodeneigenschaften und Landnutzung stellt die zentrale Größe der Bodenwasserhaushaltsmodellierung dar. Die standort- und witterungsabhängige Beschreibung des Einflusses der Vegetation in dem Modell ist hervorzuheben (s. Gudera und Morhard 2015; Blomenhofer et al. 2012).

Auf diesem Wege wurden die mittleren natürlichen Grundwasserneubildungshöhen für den Zeitraum der Jahre 1971 bis 2000 abgeschätzt (sog. Ist-Zustand in Blomenhofer et al. 2012; Abb. 5).

Im Resultat ergibt sich im Untersuchungsgebiet für die Variante I eine mittlere Grundwasserneubildungshöhe von 115 bis $120 \mathrm{~mm}$; für die Variante II beträgt sie etwa $70 \mathrm{~mm}$. Die Differenz ist erheblich. Auch die Unterschiede der Grundwasserneubildungshöhen in den Einzelflächen und deren Verteilung im Untersuchungsgebiet sind im grafischen Vergleich beider Varianten markant (s. Abb. 5). Auffällig ist der große Unterschied vor allem im südlichen Untersuchungsbereich, der das wesentliche Grundwasserneubildungsgebiet für die Einheit $\mathrm{z}+\mathrm{s}+\mathrm{mu}$ darstellt.

Für den auf Basis hydraulischer Ränder abgegrenzten Bilanzierungsraum im zentralen Bereich der Scholle 1 (Abb. 1b) konnte eine überschlägige Bilanzierung vorgenommen werden.

Die Bilanzierungsfläche beträgt etwa $91 \mathrm{~km}^{2}$. Die Teilfläche der darin ausstreichenden hydrogeologischen Einheit $\mathrm{z}+\mathrm{s}+\mathrm{mu}$ erreicht etwa $53 \mathrm{~km}^{2}$. Die mittlere jährliche Grundwasserentnahme aus diesem Bilanzierungsraum beträgt überschlägig etwa 4,7 Mio. $\mathrm{m}^{3}$.

Unter Berücksichtigung der Abflüsse der den Bilanzierungsraum erreichenden und verlassenden Gewässer, der Einleitungen in die Gewässer sowie des Randzu- und des Randabstroms lässt sich die negative Netto-Bilanzkomponente für den zentralen Bereich der Scholle 1 abschätzen.

Im Resultat erfordert die Bilanzdeckung eine mittlere Grundwasserneubildungshöhe von etwa 118 bis $134 \mathrm{~mm}$ in der hydrogeologischen Einheit $z+s+m u$. Dies entspricht etwa 6,4 bis 7,3 Mio. $\mathrm{m}^{3} / \mathrm{a}$ (Baumeister et al. 2016). 


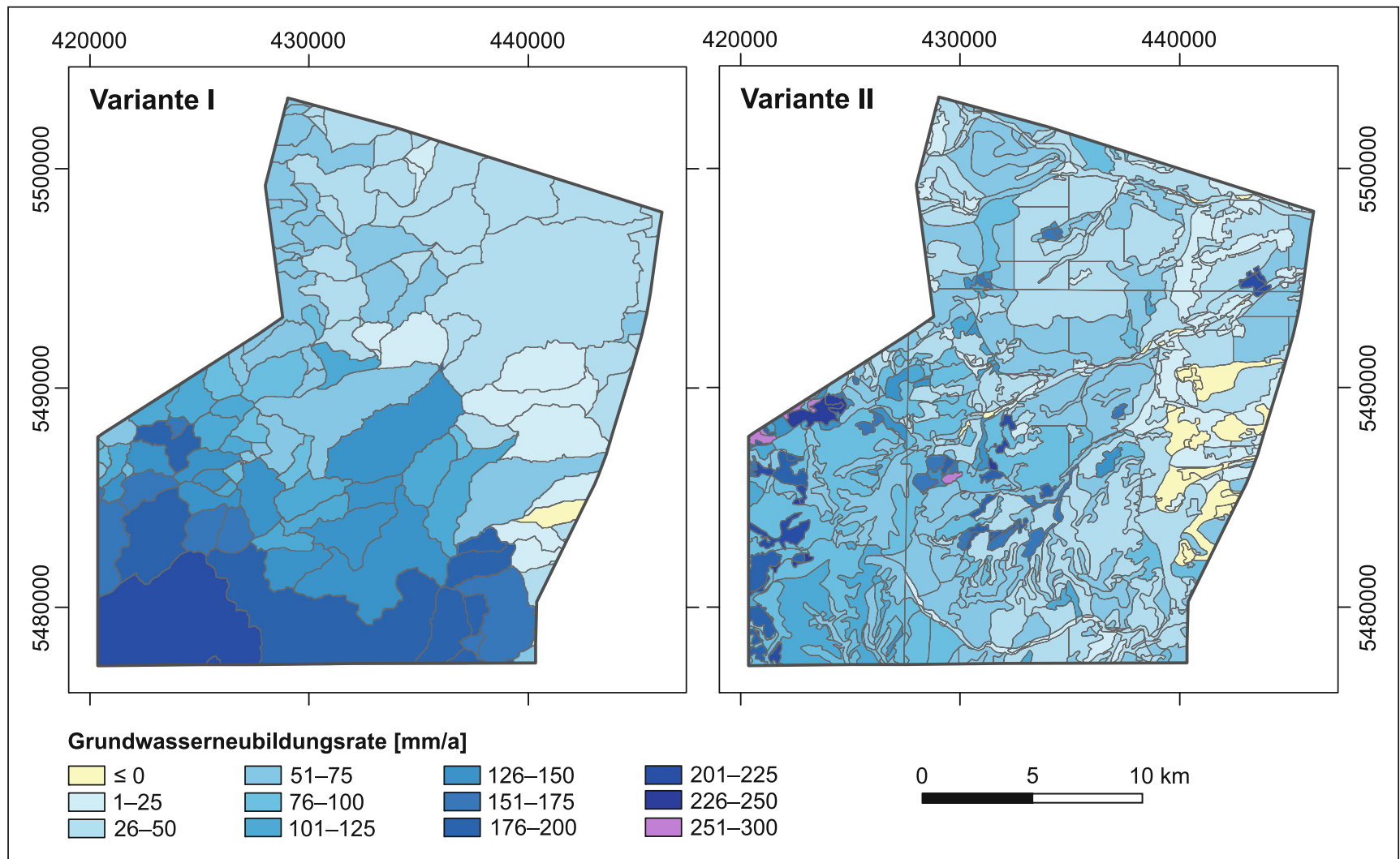

Abb. 5 Bestehende Modelle zur Grundwasserneubildung: Variante I auf Basis von Trockenwetterabflüssen (Zeitraum 1979-2008), Variante II mit dem Bodenwasserhaushaltsmodell GWN-BW (Zeitraum 1971-2000); Erläuterungen im Text

Fig. 5 Existing models of groundwater recharge: Variant I based on the low water discharge (baseflow; time period: 1979-2008), Variant II based on the model of soil water balance GWM-BW (time period 1971-2000); for details see text

Bei Ansatz des Grundwasserneubildungsmodells Variante I ergibt sich für das Bilanzierungsgebiet eine mittlere Grundwasserneubildung von $117 \mathrm{~mm}$. Sie erreicht annähernd den Wert der zur Bilanzdeckung notwendigen Grundwasserneubildungshöhe von etwa 118 bis $134 \mathrm{~mm}$. Die Grundwasserbilanz wäre damit in etwa ausgeglichen (s. a. Abb. 1b).

Für die Variante II hingegen ergibt sich für das Bilanzierungsgebiet eine mittlere Grundwasserneubildung von $91 \mathrm{~mm}$. Es bestände also eine rechnerische Bilanzunterdeckung von etwa 2,7 Mio. $\mathrm{m}^{3} / \mathrm{a}$. Bei einem angenommenen speicherwirksamen Hohlraumanteil von $1 \%$ und einer Bilanzierungsfläche der Einheit $\mathrm{z}+\mathrm{s}+\mathrm{mu}$ von etwa $91 \mathrm{~km}^{2}$ ergäbe sich - bei konstanter Grundwasserneubildung und Grundwasserentnahme, d.h. konstanter Speicherteilentleerung - eine theoretische flächenhafte Absenkung der Grundwasserdruckfläche von etwa $0,7 \mathrm{~m} / \mathrm{a}$. Diese GröBenordnung wird in den Bereichen $\mathrm{C}$ und $\mathrm{D}$ erreicht. Im Bereich A liegt sie mit etwa $0,1 \mathrm{~m} / \mathrm{a}$ darunter.

Die vorgestellten Ergebnisse der Grundwasserbeobachtungen in den Bereichen A bis D stützen die Annahme einer zumindest dort längerfristig wirksamen Speicherteilentleerung bzw. Überförderung des Grundwasserleitersystems.
Mittlerweile liegen Berechnungen zur Grundwasserneubildung für die Variante II mit Klimadaten der Jahre 2016 bis 2018 vor. Die bislang unveröffentlichten Ergebnisse zeigen dabei eine landesweite Minderung der Grundwasserneubildung gegenüber den Berechnungen für den Zeitraum 1979 bis 2008 nach Variante I um etwa $30 \%$.

\section{Zusammenfassung und Diskussion}

Die geologische Neubearbeitung mit Integration aller verfügbaren Daten zum Untergrund war die Grundlage für die Entwicklung des hydrogeologischen Modells für den Untersuchungsraum Grünstadt. Die geologisch-tektonischen Verhältnisse sind Ursache einer komplexen räumlichen Gliederung der zusammenhängenden, wasserwirtschaftlich bedeutsamen hydrogeologischen Einheit $z+s+m u$.

In den Bereichen, in denen sie das zweite Grundwasserstockwerk bildet, liegen fossile Grundwässer vor (eiszeitliche Alter). Sie nehmen nicht am meteorischen Wasserkreislauf teil.

Die Grundwasserneubildung für die Einheit $z+s+$ mu innerhalb der Scholle 1 erfolgt nur in deren zentralen und süd- 
lichen Teilbereich, in dem sie das obere Grundwasserstockwerk bildet. Aus diesem Bereich erfolgt auch die Grundwassererneuerung für die Räume, in denen diese Einheit das zweite Grundwasserstockwerk bildet, und ein erheblicher Anteil der Entnahmen stattfindet.

Die Kenntnisse zur räumlichen Gliederung der wasserwirtschaftlich bedeutsamen hydrogeologischen Einheit $\mathrm{z}+\mathrm{s}+$ mu sowie die Abschätzung der großräumigen Grundwasserströmungsverhältnisse bilden die Grundlage für die Abgrenzung bilanzierbarer Teilräume. Eine solche Abgrenzung wurde hier für den zentralen Untersuchungsbereich vorgestellt (zentraler Bereich der Scholle 1).

Für den Zeitraum etwa ab 1960 bis 2010 sind dort in den Bereichen A bis D deutliche Absenkungstrends bei den Druckhöhen sowie Veränderungen der Grundwasserbeschaffenheit in der Einheit $z+s+$ mu nachweisbar.

Die zeitlichen Verläufe sinkender Grundwasserdruckhöhen im Buntsandsteinaquifer und die Veränderungen der Grundwasserbeschaffenheit können aufgrund fehlender Daten nicht einer seit etwa 1963 möglicherweise zu hohen oder gar gestiegenen Gesamtentnahme gegenübergestellt werden. Innerhalb der Beobachtungszeiträume lassen sich die annähernd linearen Entwicklungen der Grundwasserabsenkungen jedoch als Folge weitgehend konstanter Entnahmeraten in der späten instationären Phase interpretieren. Dies weist auf eine langfristig über der Grundwasserneubildung liegende Gesamtentnahme hin. Die eingetretenen Klimaänderungen lassen zukünftig eine abnehmende Grundwasserneubildung und damit wohl zunehmende Grundwasserabsenkungen erwarten.

Es wurde eine überschlägige Grundwasserbilanzierung für den zentralen Bereich der Scholle 1 (Bilanzgebiet) vorgenommen. Auf der Basis der abgeschätzten negativen Netto-Bilanzkomponente wurde die notwendige Grundwasserneubildung abgeschätzt, die zur Bilanzdeckung notwendig ist.

Dem gegenübergestellt wurden Ergebnisse methodisch unterschiedlicher Berechnungen der Grundwasserneubildung (Varianten I und II).

Für die Variante I ist dabei eine annähernd ausgeglichene Grundwasserbilanz zu erwarten. Die Beobachtung der Druckhöhenentwicklung in den Bereichen A bis D wäre damit nicht erklärbar und müsste auf andere, eher lokale Einflüsse zurückgeführt werden. Hinweise auf solche Einflüsse liegen jedoch nicht vor.

Für die Variante II hingegen zeigt sich eine deutlich negative Grundwasserbilanz. Mit der grob abgeschätzten Speicherteilentleerung könnten die beobachteten Grundwasserabsenkungen erklärt werden. Auch die Größenordnung der Absenkungsraten ist in vergleichbar guter Übereinstimmung. Vor diesem Hintergrund erscheint das Grundwasserneubildungsmodell der Variante II als plausibler.
In jedem Fall zeigen die Untersuchungen, dass die langfristige Grundwasserbewirtschaftung vor allem eine gute Datengrundlage langfristiger Grundwasserbeobachtungen und die vollständige Erfassung wasserwirtschaftlicher Daten, vor allem der Entnahmen bedarf. Die Abschätzung der wichtigsten positiven Bilanzgröße - der Grundwasserneubildung - ist nach wie vor eine große Herausforderung; umso mehr, als sich im Zuge der Klimaveränderungen die Ableitung langjähriger Mittelwerte als zunehmend schwierig darstellen wird.

Funding Open Access funding enabled and organized by Projekt DEAL.

Open Access Dieser Artikel wird unter der Creative Commons Namensnennung 4.0 International Lizenz veröffentlicht, welche die Nutzung, Vervielfältigung, Bearbeitung, Verbreitung und Wiedergabe in jeglichem Medium und Format erlaubt, sofern Sie den/die ursprünglichen Autor(en) und die Quelle ordnungsgemäß nennen, einen Link zur Creative Commons Lizenz beifügen und angeben, ob Änderungen vorgenommen wurden.

Die in diesem Artikel enthaltenen Bilder und sonstiges Drittmaterial unterliegen ebenfalls der genannten Creative Commons Lizenz, sofern sich aus der Abbildungslegende nichts anderes ergibt. Sofern das betreffende Material nicht unter der genannten Creative Commons Lizenz steht und die betreffende Handlung nicht nach gesetzlichen Vorschriften erlaubt ist, ist für die oben aufgeführten Weiterverwendungen des Materials die Einwilligung des jeweiligen Rechteinhabers einzuholen.

Weitere Details zur Lizenz entnehmen Sie bitte der Lizenzinformation auf http://creativecommons.org/licenses/by/4.0/deed.de.

\section{Literatur}

Ad-hoc-Ag Hydrogeologie: Regionale Hydrogeologie von Deutschland. Die Grundwasserleiter: Verbreitung, Gesteine. Lagerungsverhältnisse, Schutz und Bedeutung. Geologisches Jahrbuch, Reihe A, Heft, Bd. 163. Schweizerbart'sche Verlagsbuchhandlung Nägele u. Obermiller, Stuttgart (2016). 452 S.

Baumeister, C., Kampf, J.: Materialien zur Regionalisierung des mittleren Niedrigwasserabflusses in Rheinland-Pfalz. Landesamt für Wasserwirtschaft Rheinland-Pfalz (LfW), Mainz (2002). 27 S.

Baumeister, C., Bitzer, F., Dehner, U., Franke, W.R., Schwebler, W., Wiesner, C.: Hydrogeologische Kartierung und Grundwasserbewirtschaftung Raum Grünstadt. Landesamt für Geologie und Bergbau Rheinland-Pfalz und Landesamt für Umwelt RheinlandPfalz, Mainz (2016). 94 S.

BGR: Hydrogeologische Raumgliederung von Deutschland. - Bundesanstalt für Geowissenschaften (2015). https://www.bgr.bund.de/ DE/Themen/Wasser/Projekte/abgeschlossen/Beratung/Hyraum/ hyraum_projektbeschr.html, Zugegriffen: 4. Mai 2020

Bielitz, E., Winkler, U.: Bewirtschaftung unter veränderten klimatischen Bedingungen: die sächsischen Talsperren und das Trockenjahr 2018. Energ. Wasser Praxis 12, 46-49 (2019)

Bitzer, F.: Zu den Untergrundverhältnissen im Übergangsbereich des westlichen Oberrheingrabenrandes und Mainzer Beckens. Jber. Mitt. Oberrhein. Geol. Ver. N.f. 94, 9-27 (2012)

Bitzer, F.: Zur Entwicklung der Grundwasserverhältnisse im Großraum Grünstadt, Rheinland-Pfalz. Mainz. Geowiss. Mitt. 41, 21-36 (2013)

Blomenhofer, A., Gudera, T., Neumann, J., Schwebler, W., Sprenger, W., Wingering, M.: Auswirkungen des Klimawandels auf Bodenwasserhaushalt und Grundwasserneubildung in Baden- 
Württemberg, Bayern und Rheinland-Pfalz - Untersuchungen auf Grundlage von WETTREG2003- und WETTREG2006-Klimaszenarien. KLIWA-Berichte, Bd. Heft., S. 112 (2012). Landesamt für Umwelt, Messungen und Naturschutz Baden-Württemberg (LUBW), Bayerisches Landesamt für Umwelt (BLfU), Landesamt für Umwelt, Wasserwirtschaft und Gewerbeaufsicht Rheinland-Pfalz (LUWG), Deutscher Wetterdienst (DWD)

Franke, W.R., Bitzer, F.: Beitrag zur Gliederung des nordwestlichen Teils der Rheingrabenstruktur. Mainz. Geowiss. Mitt. 47, 163-186 (2019)

Gudera, T., Morhard, A.: Hoch aufgelöste Modellierung des Bodenwasserhaushalts und der Grundwasserneubildung mit GMN-BW. Hydrol. Wasserbewirtsch. 59, 205-216 (2015)

Kampf, J., Schwebler, W.: Wasserversorgungsplan Rheinland-Pfalz Teilgebiet 6. Ministerium für Forsten und Umwelt, Mainz, S. 54 (2005)

Kampf, J., Plaul, W., Schwebler, W.: Grundwasserbericht 2007, S. 86 (2007). Ministerium für Umwelt, Forsten und Verbraucherschutz Rheinland-Pfalz
Mueef: Auswirkungen des Klimawandels auf die Trinkwasserversorgung. Ministerium für Umwelt, Energie, Ernährung und Forsten Rheinland-Pfalz, Mainz, S. 12 (2019)

Reiter, P., Sauer, T., Zimmer, M., Voigt, M.: Themenheft Klimawandel - Entwicklungen bis heute. Rheinland-Pfalz Kompetenzzentrum für Klimawandelfolgen bei der Forschungsanstalt für Waldökologie und Forstwirtschaft, Trippstadt, S. 27 (2018)

Rheinland-Pfalz Kompetenzzentrum für Klimawandelforschung: Klimawandel - Entwicklungen bis heute. Forschungsanstalt für Waldökologie und Forstwirtschaft (2019). http://www.kwis-rlp. de/de/daten-und-fakten/, Zugegriffen: 25. März 2021

Wiesner, C., Bogel, P.: Sichere Trinkwasserversorgung 2019 im mittleren Sachsen-Anhalt: Auswirkungen der Trockenheit. Energ. Wasser Praxis 12, 50-52 (2019im)

Hinweis des Verlags Der Verlag bleibt in Hinblick auf geografische Zuordnungen und Gebietsbezeichnungen in veröffentlichten Karten und Institutsadressen neutral. 